\title{
New Perspectives in the Model of Stress Response
}

\author{
I. TONHAJZEROVA ${ }^{1,2^{*}}$, M. MESTANIK ${ }^{1,2^{*}}$ \\ * These authors contributed equally to this work. \\ ${ }^{1}$ Department of Physiology, Jessenius Faculty of Medicine in Martin, Comenius University in \\ Bratislava, ${ }^{2}$ Biomedical Center Martin, Jessenius Faculty of Medicine in Martin, Comenius \\ University in Bratislava, Martin, Slovakia
}

Received February 16, 2017

Accepted February 26, 2017

\section{Summary}

The reactions of human organism to changes of internal or external environment termed as stress response have been at the center of interest during recent decades. Several theories were designed to describe the regulatory mechanisms which maintain the stability of vital physiological functions under conditions of threat or other environmental challenges. However, most of the models of stress reactivity were focused on specific aspects of the regulatory outcomes - physiological (e.g. neuroendocrine), psychological or behavioral regulation. Recently, a novel complex theory based on evolutionary and developmental biology has been introduced. The Adaptive Calibration Model of stress response employs a broad range of the findings from previous theories of stress and analyzes the responsivity to stress with respect to interindividual differences as a consequence of conditional adaptation - the ability to modify developmental trajectory to match the conditions of the social and physical environment. This review summarizes the contributions of the most important models in the field of stress response and emphasizes the importance of complex analysis of the psycho-physiological mechanisms. Moreover, it outlines the implications for nonpharmacological treatment of stress-related disorders with the application of biofeedback training as a promising tool based on voluntary modification of neurophysiological functions.

\section{Key words}

Stress $\bullet$ Homeostasis $\bullet$ Allostasis $\bullet$ Adaptation $\bullet$ Biofeedback

\section{Corresponding author}

M. Mestanik, Biomedical Center Martin, Jessenius Faculty of
Medicine in Martin, Comenius University in Bratislava, Mala Hora 4C, 03601 Martin, Slovakia. E-mail: mestanik@jfmed.uniba.sk or mestanik@gmail.com

\section{Historical background}

American physiologist Walter B. Cannon represents the first scientist who coined the term „fight or flight" to describe the integrated biological and behavioral response to threat. Cannon (1935) also described the term ,homeostasis“ as the stability of the internal environment controlled by a series of regulatory systems maintaining constancy in physiological processes - thermoregulation, glucose metabolism/regulation, water balance, and cardiovascular regulation by various pathways responding to renew balance after any perturbation. Importantly, this complex response is coordinated by the brain with the aim to maintain a set of goal values for the key internal variables (Steptoe et al. 2012).

However, the definition and concept of stress is still discussed. Hungarian endocrinologist Hans Selye (1936) defined the stress as "a non-specific response of the body to any demand for change", formulating the notion of the general adaptation syndrome. This general adaptation syndrome consists of three-stage response to exposition to stressful stimuli - alarm phase, resistance, and exhaustion (Table 1). The alarm phase is characterized by a massive alteration in regulatory processes such as activation of sympathoadrenal axis, hypothalamus-hypophysis-adrenal axis and proopiomela- 
nocortin system resulting in changes of cardiovascular parameters (blood pressure, heart rate, and blood flow), glycemia, electrolyte balance, and release of endorphins. The second phase - resistance - is characterized by mobilization of hormonal processes that increase tolerance to challenge. The third phase - exhaustion - is typical for a depletion of adaptive processes due to persisting effect of stressor with consequently increased susceptibility to a range of pathological states possibly leading to the failure of the body systems.

Table 1. The three phases of the stress response.

\section{Alarm phase}

„fight or flight“, prompt activation of: sympathoadrenal system, HPA axis proopiomelanocortin system,

$\uparrow \mathrm{BP}, \mathrm{HR}$, Glc, endorphins

\section{Resistance phase}

slower but prolonged effect of HPA

activation, energy mobilization

( $\uparrow$ Glc),

$\uparrow$ number of circulating

thrombocytes, neutrophils, inhibition

of inflammation

\section{Exhaustion}

long-term duration/excessive

intensity of the stressor, failure of adaptive mechanisms, $\uparrow$ risk of pathological states

HPA - hypothalamus-pituitary-adrenal axis, BP - blood pressure, HR - heart rate, Glc - blood glucose.

\section{Concept of allostasis and allostatic load}

The classic concept of homeostasis suggests that stability of each vital physiological function is achieved through a reactive strategy, which means that any change of the regulated parameter away from its invariant range of optimal values elicits a corrective reaction to return the variable back to the baseline level (Ramsay and Woods 2014). Thus, the principal mechanism of homeostatic regulation is represented by a coordinated system of negative feedback loops which terminates the response after restoration of homeostatic state. Importantly, the original theory of homeostasis did not include an ability of learning and anticipation of future perturbations which allows the organism to prepare for the environmental demands, activate the protective mechanisms, and reduce potential damage caused by stressors (Ramsay and Woods 2014). In other words, the homeostatic regulatory reactions are activated only if the stressor affected the physiological functions and do not persist anymore after the stress situation has ceased.

However, modern concept of stress response is based on the concept of allostasis and allostatic load (Sterling and Eyer 1988, McEwen and Stellar 1993). Specifically, the term ,allostasis“ (from Greek word ,allo“ - unstable, mutable) represents the ability to achieve stability through a change of physiological functions arising from activities of the autonomic nervous system, the hypothalamic-pituitary-adrenal axis, endocrine, cardiovascular, immune, and metabolic processes. The physiological regulatory systems are characterized by fluctuations in an integrated fashion to enable the organism to respond to the environment and to support behaviors such as eating, sleeping and exercising (Steptoe et al. 2012).

The question is what is the difference between allostasis and homeostasis? McEwen and Wingfield (2010) referred to the fact that allostatic concept adds to the concept of homeostasis. Allostasis is defined as the process of achieving stability mediated by physiological as well as behavioral alteration that is in contrast with homeostasis which maintains physiological stability by keeping the functions of the organism at the basal and fixed set point (according to Del Giudice et al. 2011). The adaptive change of physiological variables is characterized by dynamic shift in the target range of their optimal values which allows a fast adjustment to the environmental demands (McEwen and Wingfield 2010). Moreover, the ability of immediate effective response to the changing external or internal conditions is enhanced by prediction of future events and feedforward regulation of behavioral and physiological functions (Schulkin 2011). This anticipatory stress reaction is centrally driven by cortical and subcortical neural circuits which process the perception of threat and allow utilization of memory and other cognitive functions to evaluate potential risk with respect to previous experience (Stefano et al. 2008). Thus, the concept of allostasis includes the adaptive effect of learning, possible initiation of stress response without direct effect of stressor, and cognitive 
modification of the central regulatory mechanisms (Olff et al. 2005, Jezova et al. 2016, Table 2). However, evolutionary advantage of the cognitive top-down modulation of the stress response cascade may be also associated with several risks - under/overestimation of real threat, impaired executive functioning and decision making under conditions of anticipatory stress, and negative health outcomes related to activation of stress reaction even without presence of real threat (Starcke et al. 2008, Shin and Liberzon 2010).

Table 2. Differences between concepts of homeostasis and allostasis.

\begin{tabular}{|c|c|}
\hline Homeostasis & Allostasis \\
\hline $\begin{array}{l}\text { Reactive strategy - maintenance of ,normal" range of } \\
\text { physiological variables } \\
\text { Feedback regulation - stress reaction, active only in the } \\
\text { presence of stressor and terminated at restoration of } \\
\text { homeostatic state } \\
\text { Risk of negative outcomes - narrow target range of } \\
\text { regulated functions - low flexibility and adaptation, no } \\
\text { prediction of stress - slower activation of defensive } \\
\text { mechanisms }\end{array}$ & $\begin{array}{l}\text { Adaptive strategy - dynamic shift in the target range } \\
\text { of physiological variables } \\
\text { Feed-forward regulation - anticipatory stress } \\
\text { reaction, learning, cognitive modulation of stress } \\
\text { response } \\
\text { Risk of negative outcomes - under-/overestimation } \\
\text { of threat, activation of stress reaction without } \\
\text { presence of real threat, prolonged response to stress }\end{array}$ \\
\hline
\end{tabular}

The wear and tear strain which is put on the organism due to chronic or frequent activation of allostatic mechanisms has been termed allostatic load (McEwen and Stellar 1993). The concept of allostatic load summarizes the overall effect of stress on various organ systems and connects the mediators of stress reaction with pathomechanisms of certain diseases. Moreover, it allows to quantitatively assess the individual risk of stress-related disorders through evaluation of the biomarkers of allostatic load including neuroendocrine, cardiovascular, and anthropometric parameters (McEwen and Wingfield 2010). In addition to physiological correlates of allostasis, the risk of maladaptive stress response can be assessed also from psychological point of view. The psychological allostasis is characterized by individual tolerance of stress conditions termed as resilience, and a tendency towards disruption of adaptive processes termed as vulnerability (Karatsoreos and McEwen 2011).

In this context, the stress response system is characterized by significant interindividual differences in its physiological measures (e.g. baseline activation, sympathovagal dynamic balance), in the responsivity to various challenges, and it differs with distinct characteristics of psychological/social functioning and thus may further contribute to the risk for mental or somatic disorders. However, understanding of the individual specifics of the stress response system is limited (Del Giudice et al. 2011). One of the novel modern concepts concerning the meaning of the differences in stress responsivity is the Adaptive Calibration Model, as discussed in following section.

\section{Adaptive Calibration Model - new perspective of stress response system}

The Adaptive Calibration Model represents the first theory of stress development which is based on the methods of modern evolutionary and developmental biology (Del Giudice et al. 2011). Specifically, the Adaptive Calibration Model assumes that individual differences in stress response are mainly the result of conditional adaptation, i.e. the evolved organism's ability to modify its developmental trajectory to match the conditions of the social and physical environment (Del Giudice et al. 2011). This model, therefore, includes the effect of stress on physiological functioning not only in terms of acute response to stressful situation or long-term stress exposure, but also the body's ability to use the acquired information from stressful situations for adaptive processing of developmental changes with the aim of maximum possible adaptation of physiological and behavioral responses to environmental conditions (Del Giudice et al. 2011).

An example of such adaptation can be overexposure to stress in childhood followed by increased 
stress reactivity in adulthood associated with higher risk of health complaints (Heim et al. 2002). The traditional assumption is that childhood exposures to stress and adversity alter normal development in the manner of biological and behavioral dysfunctioning. In fact, both stressful and supportive environments have been part of the human experience during evolution, and thus our developmental systems have been adjusted by natural selection to respond adaptively to various challenges. It means that exposure in the stressful environments regulates development toward strategies that are adaptive under stressful conditions and protective against adverse effects in later life, and - vice versa - the well-resourced and supportive environments are associated with development of strategies that are adaptive in that context (Flinn 2006, Ellis et al. 2011a, Ellis et al. 2011b).

The Adaptive Calibration Model integrates and spreads the knowledge of several important theories concerning developmental and stress psychophysiology - polyvagal theory (Porges 1995), „tend-and-befriend“ (Taylor et al. 2000), concept of allostasis (Sterling and Eyer 1988, McEwen and Stellar 1993) and ,hawks vs. doves“" model (Korte et al. 2005). The polyvagal theory introduced a new perspective relating autonomic function to behavior that included an appreciation of autonomic nervous system as a "system", the identification of neural circuits involved in the regulation of autonomic state, and an interpretation of autonomic reactivity as adaptive within the context of the phylogenesis of the vertebrate autonomic nervous system (Porges 1995). Furthermore, the polyvagal theory emphasizes the neurophysiological and neuroanatomical distinction between two branches of the nervus vagus - older unmyelinated pathways originating from dorsal motor nucleus and myelinated pathways originating from nucleus ambiguus - and assumes that each branch supports different adaptive behavioral strategies (Porges 1995, Porges 2007, Porges et al. 2013). Especially, the social communication system, i.e. Social Engagement System is dependent on the functions of the myelinated vagus, which serves to foster calm behavioral states by inhibiting the sympathetic influences to the heart and dampening the HPA axis. Further, the mobilization system is dependent on the functioning of the sympathetic nervous system, and the most phylogenetically primitive component the immobilization system is dependent on the unmyelinated nervus vagus. These three neurobiological circuits can be conceptualized as dynamic, providing adaptive responses to safe, dangerous, or life threatening events and contexts (Porges 1995).

Taylor et al. (2000) firstly proposed a theory of female responses to stress characterized by a pattern termed "tend-and-befriend". Specifically, this theory hypothesizes that women's responses to stress are characterized by patterns that involve caring for offspring under stressful circumstances, joining social groups to reduce vulnerability, and contributing to the development of social groupings. In the aspect of this theory as a biobehavioral alternative to the fight-or-flight response, the biobehavioral attachment depends on oxytocin, estrogen, and endogenous opioid mechanisms, among other neuroendocrine underpinnings (Taylor et al. 2000). Finally, the theory of ,hawks-doves strategies" describes specific models of stress response. From the aspect of behavioral characteristics, „hawks“ are characterized by dominant, bold and aggressive pattern as ,fight-flight behavior"; in contrast, „doves“" show the ,freezing pattern“ resulting in non-aggressive and cautious behavior. With respect to physiology, while ,hawks“ are characterized by high sympathetic reactivity and reduced vagal and HPA reactivity, the „doves“ are characterized by low sympathetic reactivity associated with high parasympathetic and HPA axis reactivity (Korte et al. 2005).

Therefore, the Adaptive Calibration Model could contribute to a better understanding of the broader context of stress complex response, physiological regulatory mechanisms, personal and behavioral characteristics, and environmental impact associated with developmental aspects. In this context, stress response system is not uniform; it is characterized by individual differences contributing to four basic types of stress responsivity - sensitive, buffered, vigilant, and unemotional patterns. This aspect is discussed in the following section.

\section{Stress response system in the context of Adaptive Calibration Model}

The stress response system includes three dominant physiological regulatory systems - the sympathetic and parasympathetic branches of the autonomic nervous system, and the hypothalamicpituitary-adrenal (HPA) axis. The activity of these neuroendocrine circuits is integrated, cross-regulated, characterized by non-linear features, and they can be considered as components of a uniform functional system despite their anatomical and physiological diversity 
(Porges 1995, Adam et al. 2007, Schlotz et al. 2008, Del Giudice et al. 2011). This characteristic plays a crucial role in the main biological functions of the stress response system.

Firstly, the stress response system coordinates the organisms' physiological and behavioral responses to environmental threats involving not only unexpected/novel events, but also highly pleasurable situations (Del Giudice et al. 2011). As we discussed above, the complete organism adjustment to environmental challenge - termed as allostasis - is mediated by coordinating brain/body short-term as well as long-term changes in response to various stressors (McEwen 1998). In the terms of stressors, psychosocial stress represents just one of the major threats of today's life, and predominantly autonomic nervous system plays a key role in the physiological adjustment to psychosocial stress. Specifically, the polyvagal theory (Porges 1995, 2007) postulates that the myelinated vagus functions as an active vagal brake in which rapid inhibition and disinhibition of vagal tone to the heart can rapidly mobilize or calm an individual. Moreover, the myelinated vagus actively inhibits the sympathetic nervous system's influences on the heart and dampens HPA axis activity (Porges 2001). Functionally, the vagal brake, i.e. myelinated vagal function, enables the individual to rapidly engage and disengage with objects and other individuals and to promote self-soothing behaviors and calm states (Porges 2007).

If parasympathetic withdrawal is not sufficient to adjust in response to the psychosocial stress, activation of sympathetic nervous system provides the second mechanism of response. In particular, sympathetic activation mediates fight-or-flight reaction coordinated by the locus coeruleus as a subcortical brain structure of the noradrenergic system. From the locus coeruleus, sympathetic activation follows two pathways - direct and fast pathway through the noradrenergic innervation of visceral organs, and slower hormonal pathway via innervation of the sympathetic-adrenal-medullary axis associated with secretion of catecholamines noradrenaline and adrenaline resulting in tachycardia, tachypnoe, hyperglycemia, increased blood supply to skeletal muscles and suppression of vegetative functions (Goldstein and Kopin 2008).

Interestingly, the specific types of behavioral response to stress are characterized by noradrenergic/ adrenergic balance: high noradrenaline/adrenaline ratio is associated with proactivity and dominance, in contrast, low noradrenaline/adrenaline ratio is associated with anxiety (Netter 1991, Korte et al. 2005).

Notably, the changes in dynamic sympathovagal balance are important for assessment of individual differences in the appraisal of a given stress event reciprocal activation, i.e. sympathetic and parasympathetic activity in response to stress tends to be negatively associated with one another - parasympathetic inhibition associated with sympathetic excitation; coinhibition, i.e. low activity of both autonomic systems; coactivation, i.e. simultaneous high activation in sympathetic and parasympathetic nervous systems (Berntson et al. 1991, Alkon et al. 2003, El-Sheikh et al. 2009, Del Giudice et al. 2011).

Furthermore, the third physiological regulatory system - HPA axis - is responsible for long-term delayed response to challenge by cortisol release. Cortisol levels increase about $5 \mathrm{~min}$ after the triggering stress with a peak within 10-30 min, following with the manifestation of cortisol effects after an hour, and may be observed for several hours (Del Giudice et al. 2011). The physiological effects of cortisol are as follows: to mobilize physiological and psychological resources, e.g. energy release, alertness, vigilance, memory sensitization (Roozendaal 2000, Sapolsky et al. 2000, Flinn 2006, Tops et al. 2006, Van Marle et al. 2009, Barsegyan et al. 2010), and in part, to counter-regulate the sympathetic effects thereby facilitating the recovery (Munck et al. 1984, Del Giudice et al. 2011). Therefore, the joint effects of the sympathetic nervous system and HPA axis are synergistic in the short-term period as well as antagonistic particularly at later phases of the challenge response (Sapolsky et al. 2000, Bauer et al. 2002, Adam et al. 2007, Del Giudice et al. 2011).

Secondly, the stress response system plays a crucial role in the information encoding and filtering from the environment, thus mediating the organism's openness to environmental inputs. Specifically, the responsivity regulation can function as an amplifier, i.e. excessive reactions to the stressors with low intensity, and - vice versa - as a filter, i.e. low response to the stressors with high intensity (Del Giudice et al. 2011).

It is important to note that main neurobiological systems responsible for the information encoding and filtering include amygdala and prefrontal cortex. For example, the amygdala, which has outputs to autonomic, endocrine, and other physiological regulation systems, and becomes active during threat, is under tonic inhibitory control via GABAergic mediated projections 
from the prefrontal cortex (Davidson 2000, Thayer 2006). Thus, the default response to uncertainty, novelty, and threat is the sympathoexcitatory preparation for action commonly known as the fight or flight response for maximal survival and adaptive responses (Le Doux 1996). However, in normal modern life, this response has to be tonically inhibited via top-down modulation from the prefrontal cortex. Thus, under conditions of threat, the prefrontal cortex becomes hypoactive resulting in sympathoexcitatory subcortical centers activation essential for mobilization of energy. Notably, the several states such as anxiety or depression are characterized by prefrontal hypoactivity. In this aspect, a lack of inhibitory prefrontal functioning is related to poor habituation to novel neutral stimuli, deficits in executive function, and weak affective information processing and regulation (Thayer and Friedman 2004, Thayer 2007).

It seems that information acquired from individual divisions of stress response system depend on the specificity of individual reactions. With respect to autonomic nervous system, reduction in parasympathetic activity - vagal withdrawal - is frequent nonspecific reaction, therefore, it provides relatively little information about environmental conditions. In contrast, sympathetic activation is more specifically associated with stressors requiring fight/flight response, as indicating a potential danger or safety from environmental conditions. From this context, the most specific reaction is activation of HPA axis, which often depends on unpredictable and/or uncontrollable stressful situations associated predominantly with specific psychosocial stressors (e.g. social evaluation, family conflict, rejection/separation, and others; Del Giudice et al. 2011). For example, the frequent, high-intensity cortisol elevation in the first years of life is a likely correlate of conflictual family dynamics, insecure attachment relations and insensitive parenting (Gunnar 2005). Thus, the HPA reaction can serve as an indicator of the more serious stressful situations (Del Giudice et al. 2011).

Despite the fact that responsivity of neurobiological regulatory systems involved in stress reaction plays a crucial role in organism adjustment, the basal activity of these structures represents an important factor in individual characteristics. Specifically, the high parasympathetic activity at rest is associated with the state of positive emotionality, self-regulation, concentration, and calm conditions (Porges 1995, Porges 2007, Porges et al. 2013), the baseline sympathetic activity reflects the state of anxiety (El-Sheikh et al.
2008), and cortisol secretion indicates mobilization of energy sources (Booth et al. 2008).

\section{Patterns of stress responsivity}

The individual differences in stress responsivity arising in the Adaptive Calibration Model can be summarized as four patterns - sensitive, buffered, vigilant, and unemotional - that are characterized by basal activity and reactivity/responsivity of physiological regulatory systems - both sympathetic and parasympathetic divisions of autonomic nervous system, HPA axis; behavioral characteristics, and developmental mechanisms. It seems that the detailed knowledge of stress individual profiles could help to predict potential health as well as other adverse outcomes of the stress exposure consequences.

\section{Sensitive pattern}

With respect to autonomic physiological profile, sensitive phenotypes are characterized by high parasympathetic-linked responsivity and basal vagal tone favoring sustained and flexible attention and engagement with external environment (e.g. Beuchaine 2001, Porges et al. 2013). On the contrary, sympathetic basal activity is moderate with responsivity in the range of moderate to higher level. Thus, the activity of parasympathetic nervous system is dominant for the autonomic profile of the sensitive pattern. Moreover, the HPA responsivity is expected to be high - a highly responsive HPA promotes sensitivity to social feedback and mobilization of metabolic and psychological resources, and the high HPA responsivity also enables the organism to fast adjustment in the environmental conditions. Moreover, the HPA basal activity is moderate for this type of stress response (Del Giudice et al. 2011).

In the aspect of the behavioral characteristics, the sensitive children and adults are characterized by sufficient self-control, cognitive functioning, engagement with the environment and ability to learn (Del Giudice et al. 2011). Notably, interpersonally oriented self-control predicts increased prosocial orientation, optimism, and emotional stability, higher social skills and frustration tolerance associated with risk-taking (Uziel 2010).

From the developmental perspective, infrequent sympathetic and HPA activation in the first years of life could lead to increasing responsivity in those children with sufficient and optimal reactions to stress. Furthermore, some children are already born with 
a highly responsive pattern due to genetic predisposition and prenatal effects of maternal hormones; thus, the environment with protective factors would then maintain responsivity, whereas moderate stress would down-regulate the stress response system during the first years. Later in life, emotional stability and low level of anxiety can be assumed in sensitive adults (Belsky 2005, Belsky and Pluess 2009, Del Giudice et al. 2011).

\section{Buffered pattern}

Basal activity and responsivity of physiological systems included in stress response system are in the low-to-moderate range slightly shifted towards parasympathetic dominance reflecting the benefits and active engagement with the social environment. With respect to behavioral characteristics, the buffered children and adults are characterized by low anxiety and aggression; they are sensitive to social feedback and more oriented to long-term couple relationships. It seems that this type of buffered responsivity can be considered as a ,protective factor“ (Del Giudice et al. 2011).

In the aspect of development, buffered responsivity is predicted to arise primarily through moderate and repeated stress response system during the first years of life. Moderate stress could down-regulate responsivity in children with potentially reactive phenotype, and genetic/epigenetic factors may narrow reaction norm of some infants leading to the buffered pattern (Del Giudice et al. 2011).

It should be noted that both stress patterns - sensitive and buffered - are characterized by nonsignificant gender differences in all aspects of stress response system.

\section{Vigilant pattern}

The physiological autonomic profile is strongly associated with development. Specifically, vigilant patterns are developed in stressful context, where they allow individual to cope effectively and flexibly with dangers and threats in the physical and social environment. Therefore, the vigilant type is characterized by high basal sympathetic and HPA activity as well as high responsivity in both physiological systems. In contrast, parasympathetic basal activity and responsivity are low. However, the individuals with vigilant pattern may also display „freezing“ parasympatheticallymediated responses mediated by the dorsal vagal complex (Porges 1995, Porges 2007). Importantly, the recovery phase - period after stress exposure is prolonged, i.e. in vigilant people it may take longer time to return to baseline after sympathetic and HPA activation, and the habituation is slower (Gunnar and Vazquez 2006).

In this stress response vigilant type, behavioral patterns are represented by two gender-typical subtypes: a vigilant-agonistic pattern with dominant "fight-orflight" reaction typical for males, in which "fight" responses are dominant; and a vigilant-withdrawn pattern characterized by protective "flight" reaction typical for females. Further, the agonistic patterns should be associated with higher noradrenaline/adrenaline ratio combined with higher levels of testosterone and dehydroepiandrosterone (Van Goozen et al. 2007); however, withdrawn patterns could be characterized by a lower noradrenaline/adrenaline ratio, lower male hormones associated with higher levels of oxytocin typical for females according to „tend-and-befriend“ hypothesis (Del Giudice et al. 2011).

Gender differences are also typical for behavioral characteristics. Vigilant males are characterized by increased risk-taking, impulsivity, agonistic social competition and aggression. In females, the typical pattern includes social anxiety, lower risk-taking and impulsivity associated with fearful/withdrawn behavior (Carver et al. 2008, Tops et al. 2010, Del Giudice et al. 2011). While externalizing type of behavior is typical for vigilant male, females with vigilant pattern are characterized by internalizing type of behavior. Moreover, vigilant children who display high levels of both agonistic and withdrawn behavior may be described as a third type - the vigilantagonistic/withdrawn pattern (Rogosch and Chicchetti 1994, Del Giudice et al. 2011).

Developmental aspect expects vigilant phenotype to develop in temperamentally difficult/inhibited infants exposed to frequent and excessive stressful conditions in early life leading to up-regulation of stress responsivity. However, anxious and overprotective parenting represents a signal to the child as dangerous, unpredictable environment conducting to the development of vigilant phenotypes in temperamentally labile infants (Rubin 2002).

\section{Unemotional pattern}

This stress response „low emotivity“ pattern is characterized by physiological manner of low basal activity and reactivity in all the components of the stress response systems - autonomic nervous system and HPA axis. It means that generalized unresponsivity inhibits 
social learning and sensitivity to social feedback combined with potential increase in risk-taking by blocking information about dangers and threats in the environment. In addition, low serotonergic and dopaminergic activity are expected to be associated with unresponsive stress pattern, particularly in aggression and risk-taking (Gatzke-Kopp 2011, Del Giudice et al. 2011).

Despite the fact that decreased responsivity can be adaptive in severally stressful contexts, it is expected that especially unemotional males will be unresponsive to performance-related stressors and social evaluation, but will respond with autonomic activation to physical threats and during agonistic encounters, e.g. physical confrontations or dominance conflicts. It seems that the autonomic pattern of unemotional individuals facing agonistic threats will be complex including sympatheticparasympathetic coactivation and delayed ,fight-orflight" response (Del Giudice et al. 2011).

Further, the unemotional men exhibit a low degree of empathy and social cooperation associated with higher risk of antisocial/disruptive behavior. These specific behavioral characteristics seem to depend at least on the sex hormones, particularly antisocial behavior is associated with high levels of androgens (testosterone, dehydroepiandrosterone) coupled with low cortisol. In contrast, unemotional females are characterized by rather limited social support, aggression-linked attachment and reduction of parental behavior (Van Goozen et al. 2007, Popma et al. 2007, Booth et al. 2008, Hrdy 2009, Murray-Cloes et al. 2008, Del Giudice et al. 2011).

Two main developmental pathways leading to unemotional responsivity pattern are hypothesized. In the first pathway, an initially responsive phenotype is shifted to unresponsivity following chronic stress in childhood; for example, the typical age of onset of antisocial behavior is between 8-15 years (Kessler et al. 2005, Gunnar and Vazquez 2006, Tarullo and Gunnar 2006, Gustafsson et al. 2010). In the second pathway, the development of stress unresponsivity is linked to strong genetic predispositions. The difference between these two pathways may have important implications for the longterm development of stress responsivity, i.e. unemotional individuals with history of high responsivity can return to higher responsivity under long-term improvement of environmental conditions (Raine et al. 1997, Hawes et al. 2009, Del Giudice et al. 2011).

Concluding, the knowledge of Adaptive Calibration Model and stress responsivity could represent a major breakthrough in application to clinical practice for individual adjustment of nonpharmacological stress management. This important issue is discussed in the following section.

\section{Biofeedback - a promising tool in nonphar- macological treatment of stress-related disorders?}

Stress-related disorders (e.g. anxiety, hypertension) are related to hypersensitivity for stimulation, i.e. to neurological sensitization combined with a lowered threshold for emotional arousal. In neurophysiological context, the hippocampus, amygdala, cingulate cortex, anterior thalamic nuclei, septum and associated areas are considered as a system of integrated anatomical structures implicated in emotional expression and also in the aggregation of all sensory stimulation with affect (MacLean 1949 according to Everly and Lating 2013). Thus, sensory input is integrated and processed via limbic system and related structures that receive efferent impulses from as well as send afferent impulses to brain-stem structures - more specifically reticular activating system and locus coeruleus. In this context, the assumed neurophysiological basis of the "sensitization phenomenon" leading to hypersensitivity includes augmentation of excitatory neurotransmitters (e.g. glutamate) in limbic system, declination of the inhibitory neurotransmitters (e.g. gamma-aminobutyric acid), exchanges in the biochemical bases of neuronal activation (e.g. augmentation of phosphoproteins and/or changes of the transduction mechanism c-fos), and increased neuromuscular arousal associated with abnormal cognitive excitation (Everly and Lating 2013). Thus, the antiarousal therapy of the stress-related disorders, termed as "disorders of arousal" - such as hypertension, migraine headaches, irritable bowel syndrome, anxiety and affective disorders - could represent a natural psychophysiological phenomenon intrinsically antithetical to their underlying mechanisms. One of the promising therapeutic approaches is relaxation response including meditation, progressive relaxation, hypnosis, etc.

The concept of relaxation response is based on positive effect of limbic system desensitization, reduction of central adrenergic neurotransmission and sympathetic regulation, declination of neuromuscular arousal and cognitive excitability combined with emotional tension. It seems that the disorder of arousal might be treated effectively if limbic hypersensitivity and related factors 
could be reduced resulting in complex alteration of psychophysiological functioning, "mental calmness", and a reduction of cognitive-affective rumination (Everly and Lating 2013).

Biofeedback represents a mind-body technique in which individuals learn to voluntarily control and modify their physiology for the purpose of improving physical, mental, emotional and spiritual health. Notably, biofeedback training requires active participation of patients and often regular practice between training sessions. Therefore, biofeedback therapy is a process of training as opposed to a treatment. It means that individuals undergoing biofeedback training must take an active role and practice in order to develop the skill. Thus, rather than passively receiving a treatment, the patient is an active learner (Frank et al. 2010).

The physiological principle of the pure biofeedback application is based on operant conditioning and feedback learning. Operant conditioning is a model of learning which utilizes consequences as the means to modify the type of behavior. In biofeedback, a straight operant conditioning associated with feedback learning relies solely on the reinforcement of the signal displays in order to prompt patients to change their physiology. This biofeedback approach is used in several medical disorders such as faecal incontinence (McKee 2008, Frank et al. 2010).

However, the second biofeedback model characterized by individual approach to the patients is suitable for the patients who are suffering from a disease associated with major stress component. Specifically, this model uses psychophysiological assessment of stress complex response including a series of activities and recoveries, i.e. relax (basal) period - stress period (e.g. Stroop Color-Word test) - relaxation period after stress (recovery phase). This psychophysiological assessment of individual stress response associated with psychological evaluation to determine the thought and behavior patterns contributing to the patient's physiological vulnerability is crucial for individually-adjusted biofeedback training. In other words, the application of biofeedback training involving stress management may be the most successful therapy of the stress-related disorders (Frank et al. 2010).

Specifically, "breathing biofeedback" - training of slow and deep breathing - is the most effective method to attenuate excessive acute stress response (Everly and Lating 2013). Importantly, the sympathetic and parasympathetic nervous system is greatly affected by the process of respiration, e.g. diaphragmatic breathing stimulates the right vagus nerve facilitating full relaxation (Harvey 1978, Jerath et al. 2006). Furthermore, prolonged expiratory phase can lead to decreased neuronal firing in amygdala and hippocampus resulting in general physiological calm state associated with cognitive improvement in a manner of increased self-control and failing of obsessive thoughts (Austin 2006, Everly and Lating 2013).

\section{Conclusions}

The concepts of stress response have evolved during recent decades from the mechanistic view of homeostatic feedback regulation to dynamic models of adaptive feedforward regulatory processes with emphasis on complex evaluation of developmental, psychophysiological, social, and behavioral aspects. Novel insights from the Adaptive Calibration Model offer a rigorous theoretical background in the study of the interindividual differences in the stress responsivity, underlying physiological mechanisms and the specific risk of stress related disorders. Importantly, detailed knowledge of the organism's regulatory pathways allows the application of evidence based practice in the therapy of pathological states related to excessive exposure to stress. One of the most promising nonpharmacological methods in this field is the application of the biofeedback training strategies, which can represent a valuable tool in the treatment of stress-related diseases, particularly in the disorders of arousal. The findings summarized in this review could help to better understand the theory and possible clinical application of the novel models of stress response.

\section{Conflict of Interest}

There is no conflict of interest.

\section{Acknowledgements}

This work was supported by VEGA 1/0087/14, APVV15-0075 and the project „Biomedical Center Martin“ ITMS code: 26220220187, co-financed from EU sources. 


\section{References}

ADAM EK, KLIMES-DOUGAN B, GUNNAR MR: Social regulation of the adrenocortical response to stress in infants, children and adolescents: implications for psychopathology and education. In: Human Behavior, Learning, and the Developing Brain: Atypical Development. COCH D, DAWSON G, FISCHER KW (eds), Guilford, New York, 2007, pp 264-304.

ALKON A, GOLDSTEIN LH, SMIDER N, ESSEX MJ, KUPFER DJ, BOYCE WT: Developmental and contextual influences on autonomic reactivity in young children. Dev Psychobiol 42: 64-78, 2003.

AUSTIN JH: Zen-brain reflections. MIT Press, Cambridge, 2006. In: A Clinical Guide to the Treatment of the Human Stress Response, 3rd ed. EVERLY GS, LATING JM (eds), Springer, New York, 2013, pp 485.

BARSEGYAN A, MACKENZIE SM, KUROSE BD, MCGAUGH JL, ROOZENDAAL B: Glucocorticoids in the prefrontal cortex enhance memory consolidation and impair working memory by a common neural mechanism. Proc Natl Acad Sci U S A 107: 16655-16660, 2010.

BAUER AM, QUAS JA, BOYCE WT: Associations between physiological reactivity and children's behavior: advantages of a multisystem approach. J Dev Behav Pediatr 23: 102-113, 2002.

BEAUCHAINE T: Vagal tone, development, and Gray's motivational theory: toward an integrated model of autonomic nervous system functioning in psychopathology. Dev Psychopathol 13: 183-214, 2001.

BELSKY J, PLUESS M: Beyond diathesis stress: differential susceptibility to environmental influences. Psychol Bull 135: 885-908, 2009.

BELSKY J: Differential susceptibility to rearing influences: an evolutionary hypothesis and some evidence. In: Origins of the Social Mind: Evolutionary Psychology and Child Development. ELLIS B, BJORKLUND D (eds), Guilford, New York, 2005, pp 139-163.

BERNTSON GG, CACIOPPO JT, QUIGLEY KS: Autonomic determinism: the modes of autonomic control, the doctrine of autonomic space, and the laws of autonomic constraint. Psychol Rev 98: 459-487, 1991.

BOOTH A, GRANGER DA, SHIRTCLIFF EA: Gender- and age-related differences in the association between social relationship quality and trait levels of salivary cortisol. J Res Adol 18: 239-260, 2008.

CANNON WB: Stresses and strains of homeostasis. Am J Med Sci 189: 13-14, 1935.

CARVER CS, JOHNSON SL, JOORMANN J: Serotonergic function, two-mode models of self-regulation, and vulnerability to depression: what depression has in common with impulsive aggression. Psychol Bull 134: 912-943, 2008.

DAVIDSON RJ: The functional neuroanatomy of affective style. In: Cognitive Neuroscience of Emotion. LANE RD, NADEL L (eds), Oxford University Press, New York, 2000, pp 106-128.

DEL GIUDICE M, ELLIS BJ, SHIRTCLIFF EA: The Adaptive Calibration Model of stress responsivity. Neurosci Biobehav Rev 35: 1562-1592, 2011.

ELLIS BJ, BOYCE WT, BELSKY J, BAKERMANS-KRANENBURG MJ, VAN IJZENDOORN MH: Differential susceptibility to the environment: an evolutionary--neurodevelopmental theory. Dev Psychopathol 23: 7-28, $2011 \mathrm{a}$.

ELLIS BJ, SHIRTCLIFF EA, BOYCE WT, DEARDORFF J, ESSEX MJ: Quality of early family relationships and the timing and tempo of puberty: effects depend on biological sensitivity to context. Dev Psychopathol 23: 85-99, 2011b.

EL-SHEIKH M, ERATH SA, BUCKHALT JA, GRANGER DA, MIZE J: Cortisol and children's adjustment: the moderating role of sympathetic nervous system activity. J Abnorm Child Psychol 36: 601-611, 2008.

EL-SHEIKH M, KOUROS CD, ERATH S, CUMMINGS EM, KELLER P, STATON L: Marital conflict and children's externalizing behavior: interactions between parasympathetic and sympathetic nervous system activity. Monogr Soc Res Child Dev 74: 1-79, 2009.

EVERLY GS, LATING JM: A Clinical Guide to the Treatment of the Human Stress Response. 3rd ed, Springer, New York, 2013, $488 \mathrm{p}$.

FLINN MV: Evolution and ontogeny of the stress response to social challenges in the human child. Dev Rev 26: 138-174, 2006. 
FRANK DL, KHORSHID L, KIFFER JF, MORAVEC CS, MCKEE MG: Biofeedback in medicine: who, when, why and how? Ment Health Fam Med 7: 85-91, 2010.

GATZKE-KOPP LM: The canary in the coalmine: the sensitivity of mesolimbic dopamine to environmental adversity during development. Neurosci Biobehav Rev 35: 794-803, 2011.

GOLDSTEIN DS, KOPIN IJ: Adrenomedullary, adrenocortical, and sympathoneural responses to stressors: a meta-analysis. Endocr Regul 42: 111-119, 2008.

GUNNAR MR, VAZQUEZ D: Stress neurobiology and developmental psychopathology. In: Developmental Psychopathology, vol. 2. CICCHETTI D, COHEN DJ (eds), Wiley, Hoboken, 2006, pp 533-577.

GUNNAR MR: Attachment and stress in early development: does attachment add to the potency of social regulators of infant stress? In: Attachment and Bonding: A New Synthesis. CARTER CS, AHNERT L, GROSSMANN KE, HRDY SB, LAMB ME, PORGES SW, SASCHER N (eds), MIT Press, Cambridge, 2005, pp 245-256.

GUSTAFSSON PE, ANCKARSÄTER H, LICHTENSTEIN P, NELSON N, GUSTAFSSON PA: Does quantity have a quality all its own? Cumulative adversity and up- and down-regulation of circadian salivary cortisol levels in healthy children. Psychoneuroendocrinology 35: 1410-1415, 2010.

HARVEY J: Diaphragmatic breathing: A practical technique for breath control. Behavior Therapist 1: 13-14, 1978.

HAWES DJ, BRENNAN J, DADDS MR: Cortisol, callous-unemotional traits, and pathways to antisocial behavior. Curr Opin Psychiatry 22: 357-362, 2009.

HEIM C, NEWPORT DJ, WAGNER D, WILCOX MM, MILLER AH, NEMEROFF CB: The role of early adverse experience and adulthood stress in the prediction of neuroendocrine stress reactivity in women: a multiple regression analysis. Depress Anxiety 15: 117-125, 2002.

HRDY SB: Mothers and Others: The Evolutionary Origins of Mutual Understanding. Harvard University Press, Cambridge, 2009, $432 \mathrm{p}$.

JERATH R, EDRY JW, BARNES VA, JERATH V: Physiology of long pranayamic breathing: Neural respiratory elements may provide a mechanism that explains how slow deep breathing shifts the autonomic nervous system. Med Hypotheses 67: 566-571, 2006.

JEZOVA D, HLAVACOVA N, DICKO I, SOLARIKOVA P, BREZINA I: Psychosocial stress based on public speech in humans: is there a real life/laboratory setting cross-adaptation? Stress 19: 429-433, 2016.

KARATSOREOS IN, MCEWEN BS: Psychobiological allostasis: resistance, resilience and vulnerability. Trends Cogn Sci 15: 576-584, 2011.

KESSLER RC, BERGLUND P, DEMLER O, JIN R, MERIKANGAS KR, WALTERS EE: Lifetime prevalence and age-of-onset distributions of DSM-IV disorders in the National Comorbidity Survey Replication. Arch Gen Psychiatry 62: 593-602, 2005.

KORTE SM, KOOLHAAS JM, WINGFIELD JC, MCEWEN BS: The Darwinian concept of stress: benefits of allostasis and costs of allostatic load and the trade-offs in health and disease. Neurosci Biobehav Rev 29: 3-38, 2005.

LEDOUX J: The Emotional Brain. Simon and Schuster, New York, 1996, 384 p.

MACLEAN PD: Psychosomatic disease and the visceral brain; recent developments bearing on the Papez theory of emotion. Psychosom Med 11: 338-353, 1949.

MCEWEN BS, STELLAR E: Stress and the individual: mechanisms leading to disease. Arch Intern Med 153: 2093-2101, 1993.

MCEWEN BS, WINGFIELD JC: What is in a name? Integrating homeostasis, allostasis and stress. Horm Behav 57: 105-111, 2010.

MCEWEN BS: Stress, adaptation, and disease. Allostasis and allostatic load. Ann N Y Acad Sci 840: 33-44, 1998.

MCKEE MG: Biofeedback: an overview in the context of heart-brain medicine. Cleve Clin J Med 75 (Suppl 2): S31-S34, 2008.

MUNCK A, GUYRE PM, HOLBROOK NJ: Physiological functions of glucocorticoids in stress and their relation to pharmacological actions. Endocr Rev 5: 25-44, 1984.

MURRAY-CLOSE D, HAN G, CICCHETTI D, CRICK NR, ROGOSCH FA: Neuroendocrine regulation and physical and relational aggression: the moderating roles of child maltreatment and gender. Dev Psychol 44: 1160-1176, 2008. 
NETTER P: Do biochemical response patterns tell us anything about trait anxiety? In: Stress and Anxiety, vol. 14. SPIELBERGER CD, SARASON IG, KULCSAR S, VAN HECK G (eds), Hemisphere, Washington DC, 1991, pp 187-214.

OLFF M, LANGELAND W, GERSONS BP: Effects of appraisal and coping on the neuroendocrine response to extreme stress. Neurosci Biobehav Rev 29: 457-467, 2005.

POPMA A, VERMEIREN R, GELUK CA, RINNE T, VAN DEN BRINK W, KNOL DL, JANSEN LM, VAN ENGELAND H, DORELEIJERS TA: Cortisol moderates the relationship between testosterone and aggression in delinquent male adolescents. Biol Psychiatry 61: 405-411, 2007.

PORGES SW, MACELLAIO M, STANFILL SD, MCCUE K, LEWIS GF, HARDEN ER, HANDELMAN M, DENVER J, BAZHENOVA OV, HEILMAN KJ: Respiratory sinus arrhythmia and auditory processing in autism: modifiable deficits of an integrated social engagement system? Int J Psychophysiol 88: 261-270, 2013.

PORGES SW: Orienting in a defensive world: mammalian modification of our evolutionary heritage. A polyvagal theory. Psychophysiology 32: 301-318, 1995.

PORGES SW: The polyvagal perspective. Biol Psychol 74: 116-143, 2007.

PORGES SW: The polyvagal theory: phylogenetic substrates of a social nervous system. Int J Psychophysiol 42: 123-146, 2001.

RAINE A, VENABLES PH, MEDNICK SA: Low resting heart rate at age 3 years predisposes to aggression at age 11 years: evidence from the Mauritius Child Health Project. J Am Acad Child Adolesc Psychiatry 36: 1457-1464, 1997.

RAMSAY DS, WOODS SC: Clarifying the roles of homeostasis and allostasis in physiological regulation. Psychol Rev 121: $225-247,2014$.

ROGOSCH FA, CHICCHETTI D: Illustrating the interface of family and peer relations through the study of child maltreatment. Soc Dev 3: 291-308, 1994.

ROOZENDAAL B: Glucocorticoids and the regulation of memory consolidation. Psychoneuroendocrinology 25: 213-238, 2000.

RUBIN KH: "Brokering" emotion dysregulation: the moderating role of parenting in the relation between child temperament and children's peer interactions. In: Emotional Regulation and Developmental Health: Infancy and Early Childhood. ZUCKERMAN BS, LIEBERMAN AF, FOX NA (eds), Johnson \& Johnson, New Brunswick, 2002, pp 81-99.

SAPOLSKY RM, ROMERO LM, MUNCK AU: How do glucocorticoids influence stress responses? Integrating permissive, suppressive, stimulatory, and preparative actions. Endocr Rev 21: 55-89, 2000.

SCHLOTZ W, KUMSTA R, LAYES I, ENTRINGER S, JONES A, WÜST S: Covariance between psychological and endocrine responses to pharmacological challenge and psychosocial stress: a question of timing. Psychosom Med 70: 787-796, 2008.

SCHULKIN J: Social allostasis: anticipatory regulation of the internal milieu. Front Evol Neurosci 2: 111, 2011.

SELYE H: A syndrome produced by diverse nocuous agents. Nature 138: 32, 1936.

SHIN LM, LIBERZON I: The neurocircuitry of fear, stress, and anxiety disorders. Neuropsychopharmacology 35 : 169-191, 2010.

STARCKE K, WOLF OT, MARKOWITSCH HJ, BRAND M: Anticipatory stress influences decision making under explicit risk conditions. Behav Neurosci 122: 1352-1360, 2008.

STEFANO GB, STEFANO JM, ESCH T: Anticipatory stress response: a significant commonality in stress, relaxation, pleasure and love responses. Med Sci Monit 14: RA17-RA21, 2008.

STEPTOE A, ROSENGREN A, HJEMDAHL P: Introduction to cardiovascular disease, stress and adaptation. In: Stress and Cardiovascular Disease. HJEMDAHL P, ROSENGREN A, STEPTOE A (eds), Springer, London, 2012, pp 2-5.

STERLING P, EYER J: Allostasis: a new paradigm to explain arousal pathology. In: Handbook of Life Stress, Cognition, and Health. FISHER S, REASON JT (eds), Wiley, Chichester, 1988, pp 629-649.

TARULLO AR, GUNNAR MR: Child maltreatment and the developing HPA axis. Horm Behav 50: 632-639, 2006.

TAYLOR SE, KLEIN LC, LEWIS BP, GRUENEWALD TL, GURUNG RA, UPDEGRAFF JA: Biobehavioral responses to stress in females: tend-and-befriend, not fight-or-flight. Psychol Rev 107: 411-429, 2000. 
THAYER JF, FRIEDMAN BH: A neurovisceral integration model of health disparities in aging. In: Critical Perspectives on Racial and Ethnic Differences in Health in Late Life. ANDERSON NB, BULATO RA, COHEN B (eds), The National Academies Press, Washington DC, 2004, pp 567-603.

THAYER JF: On the importance of inhibition: central and peripheral manifestations of nonlinear inhibitory processes in neural systems. Dose Response 4: 2-21, 2006.

THAYER JF: What the heart says to the brain (and vice versa) and why we should listen. Psychological Topics 16: 241-250, 2007.

TOPS M, BOKSEM MA, LUU P, TUCKER DM: Brain substrates of behavioral programs associated with self-regulation. Front Psychol 1: 152, 2010.

TOPS M, BOKSEM MA, WESTER AE, LORIST MM, MEIJMAN TF: Task engagement and the relationships between the error-related negativity, agreeableness, behavioral shame proneness and cortisol. Psychoneuroendocrinology 31: 847-858, 2006.

UZIEL L: Rethinking social desirability scales: from impression management to interpersonally oriented self-control. Perspect Psychol Sci 5: 243-262, 2010.

VAN GOOZEN SH, FAIRCHILD G, SNOEK H, HAROLD GT: The evidence for a neurobiological model of childhood antisocial behavior. Psychol Bull 133: 149-182, 2007.

VAN MARLE HJ, HERMANS EJ, QIN S, FERNÁNDEZ G: From specificity to sensitivity: how acute stress affects amygdala processing of biologically salient stimuli. Biol Psychiatry 66: 649-655, 2009. 